\title{
A Case Report of Caries Incident in a Patient Wearing an Essix Type Retainer.
}

\author{
Albandri Alowid ${ }^{1}$, Bashayer Helaby ${ }^{1}$, and Lujain Alqarni ${ }^{1}$ \\ ${ }^{1}$ Princess Nourah bint Abdulrahman University
}

August 18, 2021

\begin{abstract}
This report presents a rare case of multiple Cl.VI carious lesions developed in a young female patient due to full-time wearing of an Essix retainer combined with poor oral hygiene and high acidic intake. The demineralization during orthodontic retention and the hygiene instructions, including the retainer cleaning protocol, are highlighted.
\end{abstract}

\section{A Case Report of Caries Incident in a Patient Wearing an Essix Type Retainer}

Albandri M. Alowid Consultant, Restorative Dental Sciences, College of Dentistry, Princess Nourah Bint Abdulrahman University, Riyadh, KSA.

AMAlOwid@pnu.edu.sa

Bashayer S. Helaby

Dental Intern, College of Dentistry, Princess Nourah Bint Abdulrahman University, Riyadh, KSA. Dr.Bashayer1415@gmail.com

Lujain M. Alqarni

Dental Intern, College of Dentistry, Princess Nourah Bint Abdulrahman University, Riyadh, KSA. lujainalgarni@hotmail.comCorresponding author:Bashayer Salem Helaby Dr.Bashayer1415@gmail.com This research is original, submitted solely to this journal, not under publication consideration elsewhere, and free of conflict of interest. All authors reviewed, approved, and consented to the submission, and they are accountable for all aspects of its accuracy and integrity in accordance with ICMJE criteria.

\section{Key words:}

Essix, retention, vacuum-formed retainer, thermoplastic retainer, Class VI caries, Incisal caries, Demineralization.

\begin{abstract}
:
This report presents a rare case of multiple Cl.VI carious lesions developed in a young female patient due to full-time wearing of an Essix retainer combined with poor oral hygiene and high acidic intake. The demineralization during orthodontic retention and the hygiene instructions, including the retainer cleaning protocol, are highlighted.
\end{abstract}

\section{METHODOLOGY:}

This report has been completed in Princess Noura bint Abdulrahman University (PNU). Institutional Review Board (IRB) Approval was taken. An appropriate patient consent form also was taken. In the form, 
the patient has given her consent for her images and other clinical information to be reported in this article. The patient understands that her name and initials will not be published. Data collected clinically, radiographically, and photographically about the patient's teeth and oral conditions. After a full examination, multidisciplinary consultations, and documentation of the case, a specific treatment plan was completed by Dr. Bashayer Helaby.

\section{PRESENTING APPEARANCE:}

A twenty-two years old, Saudi female patient came to PNU Dental clinics requesting affordable treatments to enhance her smile appearance. The patient had worn an Essix retainer full-time -Even during eating and drinking- for both arches for one year after fixed orthodontic debonding. She only removed the retainer for cleaning -using only water- once a day. The patient lost her retainer months ago before her first appointment at PNU dental clinic.

When diet counseling was performed, the patient revealed that she consumes three meals per day with a high carbohydrate rate. She also drinks three to four cups of soda per day. Low water intake also was mentioned.

Regarding the oral hygiene habits, toothbrush used with fluoridated tooth paste once a day.

Medical history showed no significant past medical issues, she was not on medications, and no allergies were detected.

Clinical findings included generalized erythematous gingiva, Class II molar classification on the right side. In contrast, the left side molar is class I (Figure 1). Multiple incisal/cusp tips carious lesions, and recurrent caries were present in the upper and lower arch (Figure 2).

Diagnoses involved plaque-induced gingivitis, multiple Class VI carious lesions related to upper and lower arches, caries assumed to be correlated to Essix retainer, multiple recurrent caries, and tooth \#13 and \#22 has necrotic pulp with asymptomatic apical periodontitis.

\section{TREATMENT:}

after a full mouth clinical examination and X-ray interpretation, the treatment plan was made. Four phases were included in the treatment plan.

The first phase was the prevention phase. Since the patient considered as a high caries risk patient, she was advised to follow a simple daily protocol that includes:

- Eliminating between-meal snacking of fermentable carbohydrates and soft drinks and replace them with nuts, vegetables, and water as much as possible.

- In the morning, after breakfast, and at night, before bed, teeth brushing using a soft brush with high fluoride $(5,000 \mathrm{ppm}$ F) toothpaste.

- Flossing one time per day after brushing at night.

- Rinse with a chlorhexidine gluconate mouth rinse $(0.12 \%)$ for one minute, the last thing at night before bed, this step only for the first week of each month.

- Daily use of a fluoride mouth rinse (0.05\% sodium fluoride).

The second phase involved periodontal treatment. Full mouth periodontal charting was performed to calculate the pockets depth as well as the plaque and bleeding index. After that, full mouth supragingival scaling was completed. Two weeks after, a follow-up appointment was scheduled. The gingival health was significantly improved.

The Operative phase was the third phase. First, primary impressions were taken to study the case and make a wax-up for a smile analysis. After that, a Mock-up appointment was made to evaluate the pronunciation and estimate the smile appearance. New primary impressions were taken to fabricate new diagnostic casts.

Then, Endodontic treatments were established for tooth \#13 and \#22. 
After root canal treatment, the teeth were closed with Glass Ionomer Cement. Subsequently, caries excavation and direct composite restorations were placed to restore the cavities in both arches. (Figure 3-4) Furthermore, two All Ceram crowns were placed to restore tooth \#13 and \#22. The patient's oral health generally was enhanced. The smile appearance was significantly improved.

The fourth phase is the follow-up phase. The recall visits scheduled every:

3-4 months for examination and fluoride varnish application.

6-12 months for Bitewing films until no cavitated lesions are showed.

\section{DISCUSSION:}

To prevent relapse after orthodontic treatment and maintain the gained results over a long time, the retention phase is critical (Ramazanzadeh et al. 2018).

Orthodontists consider the Hawley retainer as a gold stander in retention (Jolley 2016). But nowadays, vacuum-formed removable retainers or thermoplastic types of retainers, which are commercially named Essix, are widely used for many reasons (Ab Rahman et al. 2016). Being invisible, comfortable, and with short manufacturing time and a reasonable price makes Essix appliance an excellent choice to the patients ( $\mathrm{R}$ et al. 1995). Essix appliance is first introduced by Sheridan et al. as an alternative to the traditional retainers (Sheridan and LeDoux 1993). Essix retainer is made from polyvinyl siloxane sheets that take the anatomical shape of the teeth (Ramazanzadeh et al. 2018). Another study reported that there are three types of materials that Essex retainer could be made of. The first material is a copolyester that made type A Essix. Polypropylene or ethylene copolymer is the second material, and it is used to made Essix retainer type C. Essix type A is more aesthetic than type C because of better clarity. However, type A tends to crack and tear. Type C Essix is stronger than type A but with low mechanical retention. After a while, manufacturers created the third type of materials, combining the previous two types. The third material gathers all the advantages of type A and C. Eventually, these new material shows less durability and more cracks and a high ability to discoloration (Rinchuse et al. 2007).

Another study presented that Essix materials used for the thermoplastic retainers are the following: polypropylene polymer-based material, polyethylene copolymers, and polyethylene terephthalate glycol copolymer (a thick sheet material). Regularly, the thicknesses of Essix between $0.75 \mathrm{~mm}$ and $1 \mathrm{~mm}$ (Vaida et al. 2020).

Though the design of Essix retainers is more comfortable than conventional retainers, Essix should not be used for an extended period, and the patient should follow a specific protocol. (Ramazanzadeh et al. 2018). In this case report, the patient had been using upper and lower Essix retainer full-time for one year, following the completion of fixed orthodontic therapy. Some of the recommended protocols for thermoplastic retainers are full-time wear for the first one week and then night-time only for at least one year. For better incisor alignment in the mandible, the patients should be advocated to wear Essix full-time for four months and then at night only (Ramazanzadeh et al. 2018).

Many changes can occur in the oral environment when a patient is wearing removable orthodontic retainers. Our patient was eating her meals without removing the retainer. We assumed that this repeated scenario was the main reason behind the formation of caries. A study showed that the retainers introduce additional surfaces that biofilms may form on. Adolescent orthodontic patients are more caries-prone papulation due to biofilm formation. These biofilms can induce caries-causing bacteria that could migrate to tooth surfaces and accelerate caries' formation (Jolley 2016). Also, a previous study reported a high amount of Streptococcus mutans (S. Mutans) in children using removable appliances (Batoni et al. 2001). Essix should be used after cleaning both the inner surface of the retainer and the teeth surfaces. While the retainer works as a cover that prevents saliva from washing teeth, colonization of S. Mutans and Lactobacillus on dental surfaces increases (Türköz et al. 2012). The bacteria adhere to the teeth, break down carbohydrates in food/beverage remnants, and produce acid. The acidic environment with time leads to demineralization of the teeth surfaces 
that in contact with the retainer's inner surface, i.e., the cusp tips of the posterior teeth and the incisal edges of anterior teeth. G.V. Black classifies this caries pattern as class VI.

Research on oral hygiene instructions represents a reduction in S. Mutans by $99 \%$ with brushing. The same study recorded three effective methods to clean the Essix retainer: the first one is brushing with fluoride toothpaste, the second is brushing with chlorhexidine gel, while the third method is the immersion of the retainer in a chlorhexidine solution. There were no statistically significant differences between any cleaning groups for any microorganisms except in the group that uses CHX mouthwash and gel to eliminate the microorganism more effectively than the fluoridated toothpaste. Therefore, brushing with fluoride toothpaste can be approved as an effective method for cleaning retainers in most cases. In patients where bacterial infection must be avoided because of immunosuppression or any other disease, chlorhexidine gel or mouthwash is suggested (Chang et al. 2014).

\section{CONCLUSION:}

Even though oral hygiene is the patient's responsibility, educating the patients is the dental staff's responsibility. Orthodontists, general dentists, and hygienists are in charge to:

- Give definite instructions about the Essix retainer wearing duration.

- Give an exact cleaning protocol for both teeth and retainer.

- Advocate the patients to attend monthly follow-up appointments for the first six months.

- For patients with poor oral hygiene or multiple incipient carious lesions, oral hygiene improvement should be observed while using Hawley retainer; then, the Essix retainer could be used.

\section{ACKNOWLEDGMENT:}

The authors thank the Department of Research and Innovation, Collage of Dentistry, PNU.

The funding agencies have no role in the submitted work.

\section{AUTHOR CONTRIBUTIONS:}

A. Alowid, B. Helaby, and L. ALQarni contributed to the conception, design, data acquisition, and interpretation, and critically revised the manuscript. All authors gave their final approval and agreed to be accountable for all aspects of the work.

\section{CONFLICT OF INTEREST:}

The authors declare no potential conflicts of interest with respect to the authorship and/or publication of this article.

\section{FIGURE LEGEND:}

Figure 1: Lateral and frontal view, before the treatment.

Figure 2: Occlusal view, before the treatment.

Figure 3: Lateral and frontal view, After the treatment.

Figure 4: Occlusal view, After the treatment. 

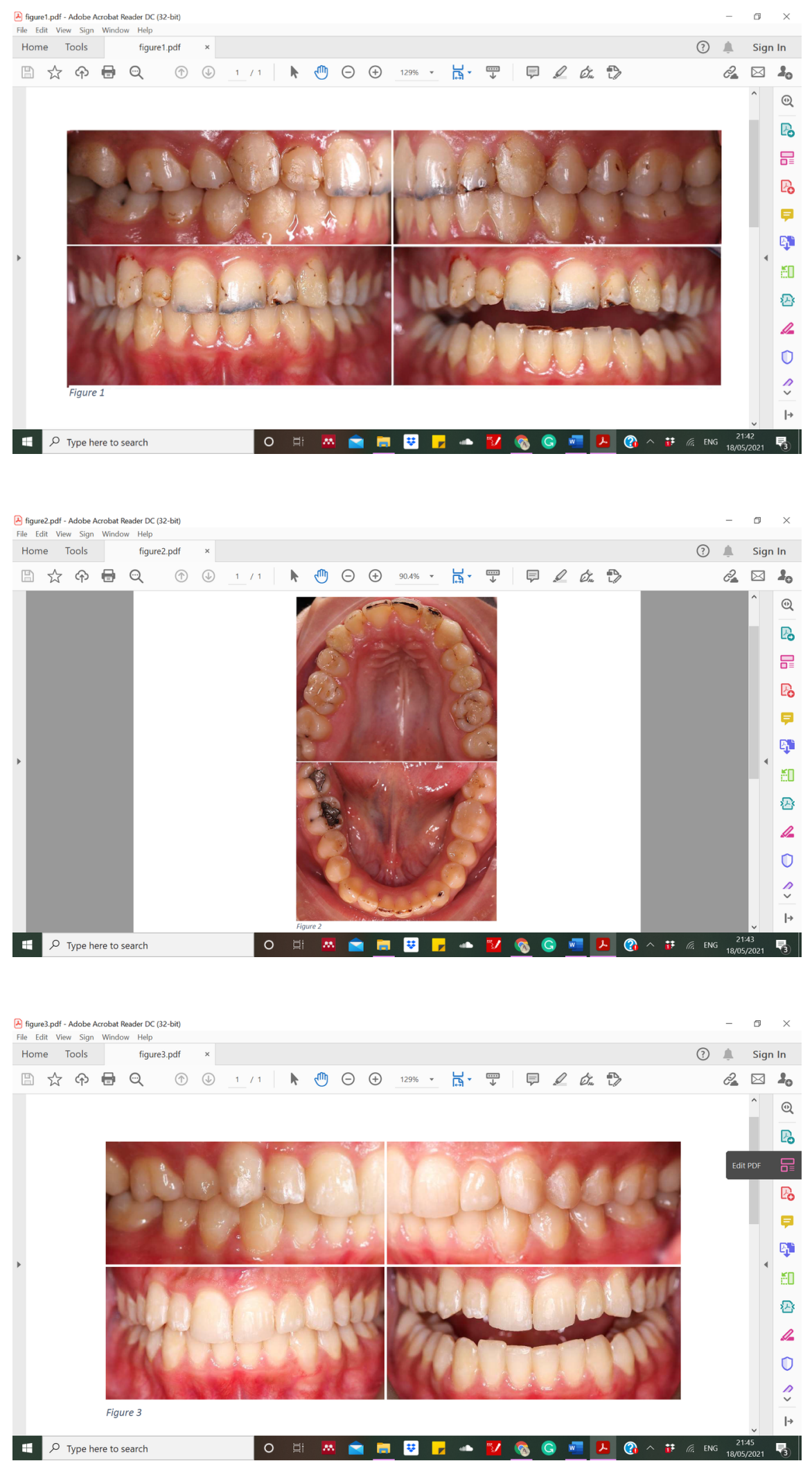


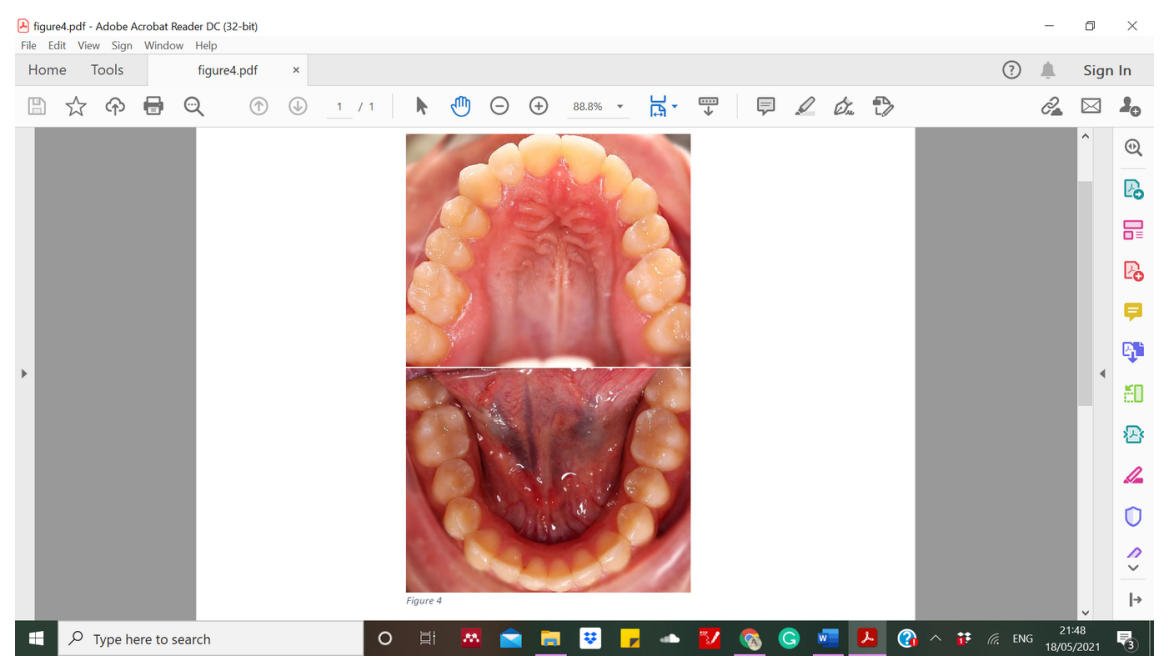

\section{REFERENCES:}

1. Ab Rahman N, Low $\mathrm{T}$, Idris N. 2016. A survey on retention practice among orthodontists in Malaysia. The Korean Journal of Orthodontics . 46(1), p.36.

2. Batoni G, Pardini M, Giannotti A, Ota F, Rita Giuca M, Gabriele M, Campa M, Senesi, S. 2001. Effect of removable orthodontic appliances on oral colonisation by mutans streptococci in children. European Journal of Oral Sciences . 109(6), pp.388-392.

3. Chang C, Al-Awadi, S, Ready D, Noar J. 2014. An assessment of the effectiveness of mechanical and chemical cleaning of Essix orthodontic retainer. Journal of Orthodontics . 41(2), pp.110-117.

4. Jolley D. 2016. The Effects of Orthodontic Appliance Base Plate Material, PMMA, Infused with Silver and a Novel Antibacterial Compound On Biofilm FormationUNLV Theses. Dissertations, Professional Papers, and Capstones. 2868.

5. Ramazanzadeh B, Ahrari F, Hosseini Z. 2018. The retention characteristics of Hawley and vacuumformed retainers with different retention protocols. Journal of Clinical and Experimental Dentistry . pp.0-0.

6. Rinchuse DJ, Miles PG, Sheridan JJ. 2007. Orthodontic retention and stability: a clinical perspective. $J$ Clin Orthod . 41(3):125-32.

7. R A, Sampson P, Little R, Årtun J, Shapiro P. 1995. Long-term changes in arch form after orthodontic treatment and retention. American Journal of Orthodontics and Dentofacial Orthopedics . 107(5), pp. $518-530$.

8. Sheridan JJ, LeDoux W. 1993. Essix retainers: fabrication and supervision for permanent retention. McMinn R J Clin Orthod . 27(1):37-45.

9. Türköz, Ç, Canigür Bavbek, N, Kale Varlik S. and Akça G. 2012. Influence of thermoplastic retainers on Streptococcus mutans and Lactobacillus adhesion. American Journal of Orthodontics and Dentofacial Orthopedics . 141(5), pp.598-603.

10. Vaida L, Bud E, Halitchi L, Cavalu S, Todor B, Negrutiu B, Moca A, Bodog F. 2020. The Behavior of Two Types of Upper Removable Retainers-Our Clinical Experience. Children . 7(12), p.295. 


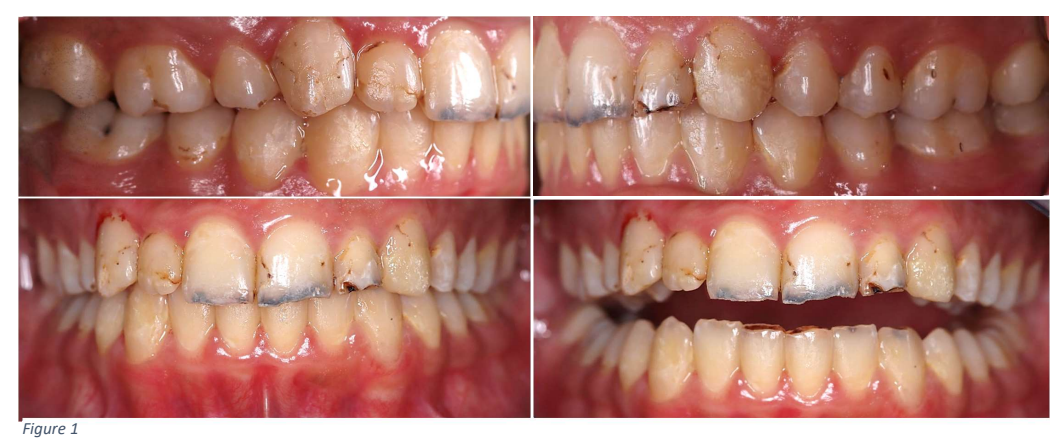




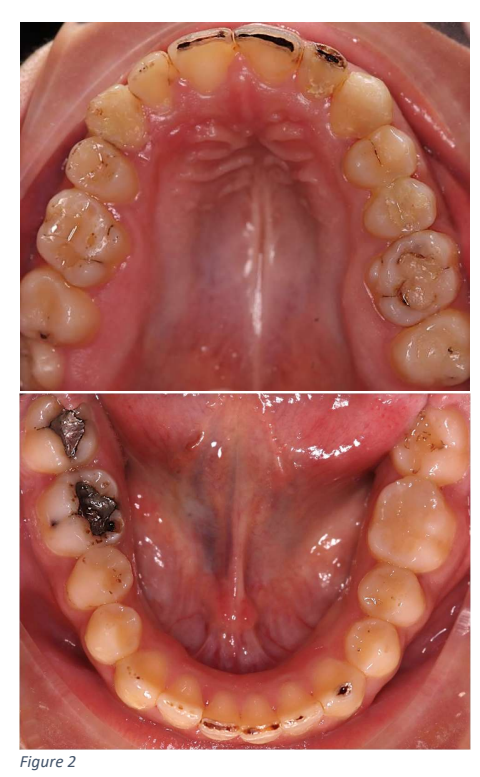




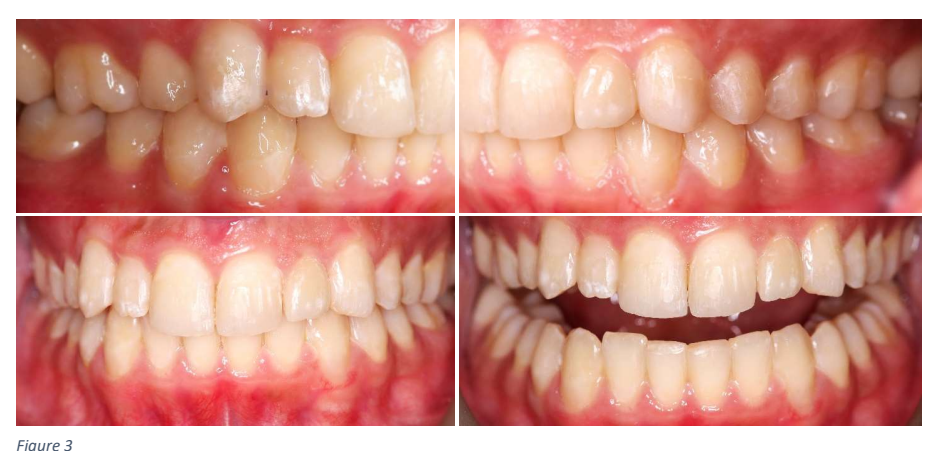




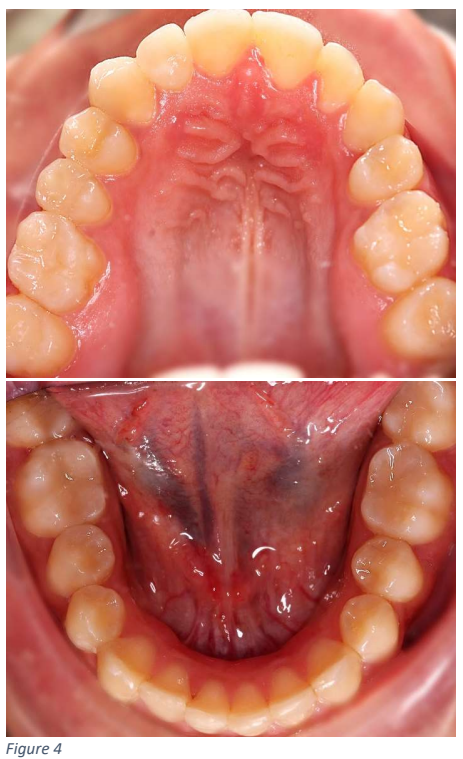

